\title{
X-ray Holography with Atom Resolution
}

\author{
G. Faigel, M. Tegze \\ Research Institute for Solid-State Physics, Budapest
}

A new concept based on well-established fundamental principles allows the high-resolution holographic imaging of atoms inside bulk cystals using X-rays.

Throughout history humans have been fascinated by the microscopic world, the world which is hidden from the naked eye. This led to the discovery of the light microscope in the 16th century, the electron microscope in the 1940 s and today's high-resolution imaging devices, the tunnelling and atomic force microscopes. While providing direct, magnified images with atomic resolution of objects in real space, the latter only give information about surfaces.

The discovery of X-rays 101 years ago opened up a way to see into the bulk. A variety of diffraction techniques have been developed to allow us to study atomic arrangements. However, diffraction methods work in reciprocal space which means the measured data have to be transformed to obtain real-space atomic coordinates. This procedure is not straightforward, because when we collect diffraction data we measure the intensity of the scattered beam but we cannot measure phases. This loss of phase information has to be substituted by some additional knowledge.

There are many ways to circumvent this problem. We can usually acquire the extra knowledge from additional, independent measurements. However, the questions remain: Is there a method which measures phases and intensities simultaneously? Second: Is there a simple, straightforward way to reconstruct the three-dimensional arrangement of objects in real space? Positive answers were given by Dénes Gábor about 50 years ago when he invented holography [1].

Holography is used nowadays in science, technology and everyday life. Its principle is very simple: when a coherent beam illuminates an object, part of the beam is scattered (object wave), and part reaches the detecting surface without

Gyula Feigel and Miklos Tegze are senior scientists with the KFKI Research Institute for Solid-State Physics, Budapest. interaction (reference wave). These two waves interfere and the interference pattern is recorded. There are many ways to capture a hologram. In every case the important item that we have to know is the reference beam, because to recover the objects in three dimensions the hologram has to be illuminated with the inverse of the reference beam. Mathematically, this is equivalent to an integral transformation which allows a numerical reconstruction using a computer

The most serious problem in applying holography to atomic imaging is the resolution. The resolution is limited by the wavelength and the source size or detector resolution. For a long time, coherent sources were only available for the visible light range. This limited resolution to micron or larger distances. With the introduction of coherent radiation from undulator devices at synchrotrons the wavelength limit was pushed down to the soft $\mathrm{X}$-ray regime of $100 \AA$ or thereabouts.

However, we cannot attain a resolution of $100 \AA$ owing to two other limiting factors, i.e., the source size and detector resolution. At the present level of technology the resolution is somewhere in the range of $500-1000 \AA$. An improvement of some three orders of magnitude is needed to reach atomic resolution. A new concept has been developed to bridge this gap.

\section{X-ray Holography}

About 10 years ago Abraham Szoke suggested [2] that the individual atoms present in the sample should be used as sources of radiation (the so-called inside source concept). How would this work? For simplicity let us consider only two atoms within a sample. The source emits a spherical wave which can be scattered by the neighbouring atom, or it can reach the detecting surface without interaction. A hologram is formed by the interference of these two waves (Fig. 1a). Of course, in practice there are $10^{23}$ or more atoms in the sample so some conditions have to be satisfied to obtain a single hologram, namely: - the environment of every source atom has to be the same, and oriented in the same way;

- the emitted radiation have to be monochromatic;

- the waves emitted by different atoms have to be incoherent;

- the size of the sample has to be much smaller then the sample - detector distance. Under these conditions, separate but identical holograms are simply added.

All of these conditions can be satisfied using a single-crystal sample. Szoke's idea was realized experimentally for the first time in 1990 using electrons as hologramforming waves [3]. Harp and coworkers subsequently reconstructed the threedimensional atomic order of the surface of a $\mathrm{Cu}$ single crystal. However, we were almost back where we started with this real-space information since one only obtains three-dimensional atomic order in a surface region some 10-30 atomic layers thick. To see into the bulk we have to combine X-rays with the inside-source concept. It turns out that the problem of an internal source (e.g., a fluorescent atom) located on a crystallographic site has been solved long ago: the result is the Kossel line pattern [4].

Kossel lines are analogous to Bragg peaks except that in the Bragg case we have an external source and in the Kossel case we have an internal source. The highintensity surfaces are cones centred on the

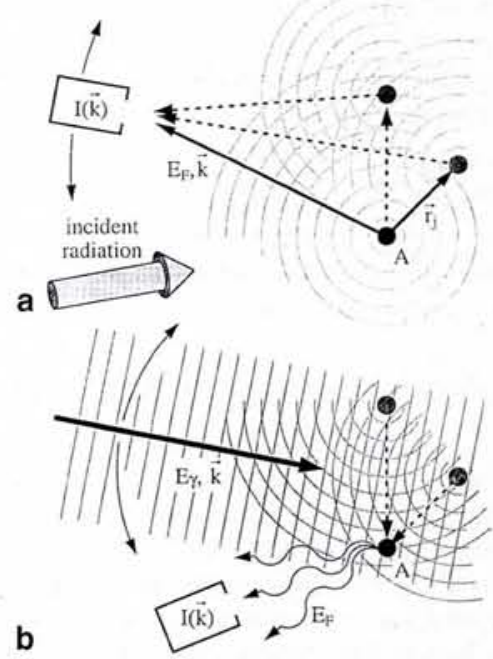

Fig. 1. Schematic illustration of $X$-ray hologram formation. a: Internal source; atoms (A) in the sample excited by an external source of $X$-rays emit a spherical wave. This can be scattered by the neighbours or go directly to the detector. $\mathbf{b}$ : Internal detector; $X$-rays incident on the sample can reach the detector atom (A) directly or after scattering on the neigbours. From [8]. 
source atom and their opening angle is determined by Bragg's law. Kossel line patterns were first recorded in 1935 [5]. At this point it might seem that one merely has to reinterpret the Kossel patterns as holograms to reconstruct the three-

dimensional image of atoms in a crystal. However, a more careful analysis reveals that the sharp Kossel lines are a consequence of the crystal's long-range translational periodicity [6]. So atoms at large distances apart contribute significantly to the pattern, which cannot then be backtransformed using the usual holographic reconstruction.

On analyzing the angular frequency components of the interference pattern as a function of the distance from the source atom it turns out that the high-frequency components are coming from distant atoms, and low-frequency components from near-neighbour atoms [6]. A lowpass filter allows one to filter out the contribution of distant atoms. This process eliminates the Kossel lines leaving only the holographic information of the nearneighbour atoms. Numerical simulations have shown that the variation of the intensity caused by the holographic effect is small (in the range of one-tenth of a percent) but within the reach of presently available measurement techniques [6].

\section{The First Imaging Experiment}

This result prompted us to construct a setup for measuring an X-ray hologram [7]. For practical reasons we chose a $\mathrm{SrTiO}_{3}$ single-crystal platelet to demonstrate the principle. First, the wavelength of the $\mathrm{Sr} \mathrm{K} \alpha$ line of about $0.8 \AA$ allows an adequate resolution. Second, $\mathrm{Sr}$ atoms can be excited efficiently by Mo $\mathrm{K} \alpha$ radiation which is readily available at conventional laboratory sources. Third, in $\mathrm{SrTiO}_{3}$ the $\mathrm{Sr}$ atoms are located on a simple cubic lattice, thereby enabling a straightforward and clear interpretation of experimental data.

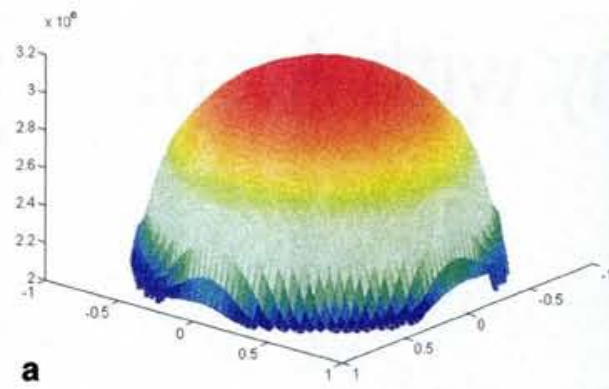

The raw data for the first experiment are shown in Fig. 2a. No holographic oscillation can be seen since it is suppressed by the combined effect of absorption and the flat-plate geometry. A very careful subtraction of the $40 \%$ change caused by absorption has to be carried out to see the holographic intensity variation of $0.3 \%$ (Fig. $2 b)$. Since the surface of the crystal was parallel to the (100) crystallographic plane the hologram should reveal the four-fold symmetry which was assumed for the reconstruction. The reconstructed image is depicted in Fig. $2 c$ for three planes parallel to the crystal's physical surface. For clarity, the edges of the elementary unit cells are shown as white lines. Note that only the $\mathrm{Sr}$ atoms are resolved owing to the small scattering factors of $\mathrm{Ti}$ and $\mathrm{O}$ atoms compared to $\mathrm{Sr}$ atoms.

After these results were published a US/German group reported holographic imaging of atoms using a modified version of the experiment described above [8]. In this case the fluorescing atoms were used as detectors and not as sources, and the hologram was formed by the incident beam (the experiment is illustrated schematically in Fig. 1b). The method should in principle work the same way as our experiment even though the first results only a gave a two-dimensional crosssection of one plane (the plane parallel to the crystal surface and containing the detector atom) and not a reconstruction of the atomic positions in three dimensions.

\section{Applications of X-ray Holography}

The value of an experimental method is largely determined by its applicability. It is therefore appropriate to mention some potential applications of X-ray holography, even though the first demonstration experiments have only just been performed. Unambiguous knowledge of the atomic environment of a specific element in a crystal can greatly facilitate the determination of crystal structure from traditional diffraction data. Since crystallographers are working on increasingly complex structures having information about atomic positions in three dimensions should be a great help. Furthermore, there are systems for which unique information could be gained from holography. These include: crystals with implanted atoms, systems having buried interfaces, nonperiodic but orientationally ordered materials, etc. These applications rely on the straightforward application of presently available techniques. However, we can look a bit further into the future. The application of high-intensity synchrotron sources (see page 182) together with a fast-detector system might allow time-resolved studies in the millisecond range. By combining this with a high-speed computer and an advanced evaluation procedure one can follow slow atomic motions in real time. Another possibility is the use of nuclei as sources of radiation. Since many nuclear gammatransitions are sensitive to hyperfine fields in the sample, holograms formed by waves originating from nuclear decay could give information on the magnetic order or electric field gradient present in materials.
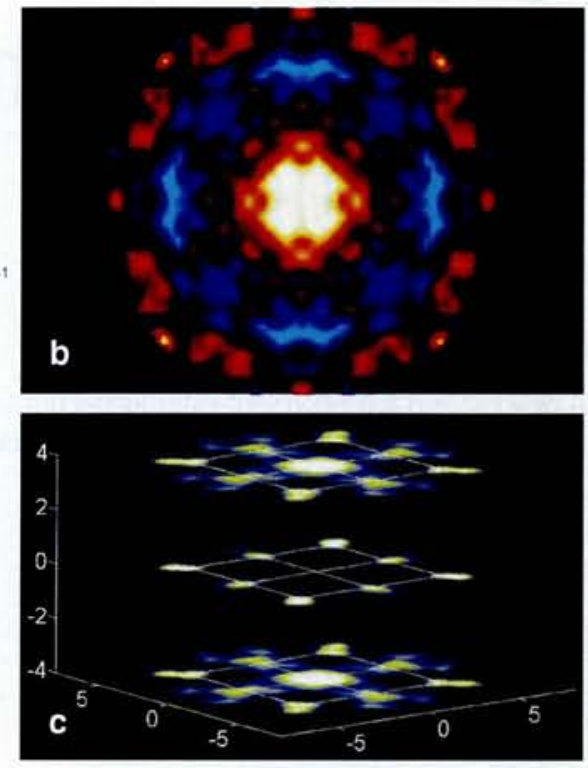

Fig 2. X-ray holograpic imaging of atoms in $\mathrm{SrTiO}_{3}$. a: Raw intensity data for the determination of the hologram. The $40 \%$ change in intensity is caused by absorption and the flat-plate geometry used in the experiment. b: The hologram of (a) after absorption correction and subtracting the contribution of the direct beam. The variation of intensity between high and low intensity points is $0.3 \%$. c: Three reconstructed planes (transformed from (b) in real space showing the $\mathrm{Sr}$ atoms. The outlines of eight fundamental unit cells are shown as solid lines; the scale is given in Angströms.

But the method has experimental advantages since one can easily change the wavelength of the hologram-forming radiation. So it it possible to decrease the effect of a Fourier transformation over a finite range (spurious oscillations) and the cancellation of intensities arising from twin images (artifacts of holographic reconstruction that can distort the intensities of atoms in a centrosymmetric crystal).

Many applications (see insert) of Xray holography with atom resolution can be thought of for a wide community, but our aim is to develop the basic experimental technique and evaluation procedures for the general user.

\section{References}

[1] D. Gábor, Nature (London) 161 (1948) 777.

[2] A. Szike, in Short Wavelength Coherent Radiation: Generation \& Applications, Eds.: D.T. Attwood \& J. Boker, AIP Conf. Proc. No. 147 ( AIP, New York, NY; 1986 ) 361.

[3] G.R. Harp, D.K. Saldin \& B.P. Tonner, Phys. Rev. Lett. $65(1990) 1012$.

[4] M. von Laue, Ann. der Physik 23 (1935) 705.

[5] W. Kossel, V. Loeck \& H. Voges, Zeit. f. Physik 94 (1935) 139.

[6] M. Tegze \& G. Faigel, Europhys. Lett. 16 (1991) 41.

[7] M. Tegze \& G. Faigel, Nature 380 (1996) 49. [8] T. Gog et al., Phys. Rev. Lett.76 (1996) 3132.

\section{Acknowledgment}

This work was supported by grant AKP 96-225/12. 